\title{
STUDY ON CONTOROL POINTS LAYPUT BASED ON OBLIQUE PHOTOGRAPHY
}

\author{
Liu Weiwei ${ }^{1}$, Luo Guangfei ${ }^{1}$, Cai Zhigang ${ }^{1}$ \\ ${ }^{1}$ Zhejiang First Surveying and Mapping Institute, Hangzhou 310012, China
}

KEY WORDS: Oblique Photography, Layout of Control Points, Density of Control Points, Precision of Control Points

\begin{abstract}
:
In practical projects, suitable high-precision control points are often added in the adjustment calculation so as to accurately recover the relationships between different light beams at the time of photography and finally obtain the results of high-precision oblique photography data. This paper studies the layout of control points of oblique photography data, and provides certain theoretical and practical guidance to the data processing application of actual oblique photography projects under the circumstance that there is no corresponding specification as the guidance for data processing of oblique photography at present.
\end{abstract}

\section{ITRODUCTION}

It can be found that although most post-processing of oblique photography data starts from computer vision, computer graphics, pattern recognition and other perspectives, from the view of essence, their basic principle is still the computation with the collinearity equation. Therefore, according to traditional photographic survey, the recovery precision of elements of exterior orientation of oblique photography still relies on the POS data and control points. Generally, it is difficult for the POS systems equipped on aerial photography platforms to meet the requirements of high-precision surveying and mapping, or no IMU system is equipped on some UAV platforms from the perspective of costs and flight efficiency; therefore, suitable high-precision control points are often added in the adjustment calculation so as to accurately recover the relationships between different light beams at the time of photography(Wang Jing, etc.2011) and finally obtain the results of high-precision oblique photography data(Fan Panfeng, Li Lulu,2017). In order to meet the actual production, it is necessary to do a correspon ding research on the requirements for the layout of control points in oblique photogrammetry. In this paper, control point density and precision experiments are conducted on the oblique data of the test area to obtain the accuracy that can be achieved under different layout conditions of control points. This provides theoretical and practical guidance for data processing application of actual oblique photography projects.

\section{INFLUENCE OF CONTROL POINTS ON BASED ON THE PRECISION OF AERIAL TRIANGULATION OF OBLIQUE PHOTOGRAPHY}

\subsection{Research on the Development of Layout of Control Points in Photogrammetry}

In traditional aerial photogrammetry, the layout of control points is based on the corresponding specification (Deng Fang, Zhang Jun, etc.2011). In the era for film aerial photography, the theoretical basis and basis of the accuracy estimating formula for the layout of control points is the formula for estimating the most vulnerable accuracy of single polynomial adjustment. The corresponding standard layout scheme is mature and stable, and it is proved to be effective and reliable after long-term production practice. In digital aerial photography era, the method of beam and regional network adjustment is used to carry out the aerial triangulation, and the corresponding layout scheme of control points is analyzed theoretically under the ideal square region condition, but that's not the case in the actual operation. Therefore, at present, the layout scheme of control points for digital aerial photography does not apply to all aerial photography situations, Usually, it is based on the precision estimation formula of the navigation strip method to inversely seek the image control point scheme and appropriately relaxes, and then the project production can be conducted after the necessary experiment verification.

Since the development of oblique photography, there is no corresponding external control specification as the guidance of actual operation. The main reason is that the camera for oblique photography is extremely diversified, and the region is not regular. Besides, the large overlap and great angle of oblique images have gone beyond the traditional aerial photography category. Therefore, there is no suitable theoretical basis for the layout of control points. The acquisition of remote sensing data with large overlap, high resolution and high redundancy in oblique photography can achieve simultaneous processing of multiple images, and the increased excess observations can greatly reduce the uncertainty of matching, and can improve the accuracy of image matching. Therefore, the commonly layout scheme of control points in oblique photography is to ensure the placement of the four corners, and then the point layout is performed in units of hundreds of meters. The research work of this paper is based on this.

\subsection{Influence of the Density of Control Points on the Precis- ion of Aerial Triangulation of Oblique Photography}

From the perspective of practical production, this paper selected UVA oblique photography data as an experiment source in a region of southern china. The topographic feature was flat terrain, the flying height was about $180 \mathrm{~m}$ with a 5-lens camera, the image resolution was better than $3 \mathrm{~cm}$, and the image size is $6000 * 4000$. The experiment data involved 3 flights, the number of images was 12200 and the area was about $2.2 \mathrm{~km}^{2}$. The experiment set the space of distance of control points in $700 \mathrm{~m}$, $500 \mathrm{~m}$ and $250 \mathrm{~m}$ separately to test the density of control points. The distribution of control points and check points can be illustrated in the diagram, where yellow cross is marked as control points and red circle marked as check points. 


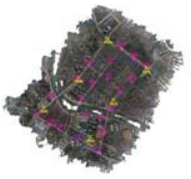

$250 \mathrm{~m}$ pan

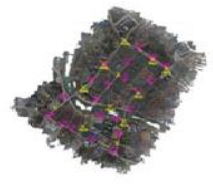

$500 \mathrm{~m}$ pan

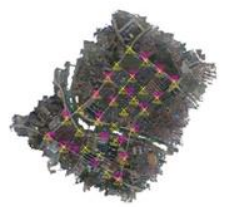

$750 \mathrm{~m}$ pan
Figure 1. Control Points and Check Points Distribution Diagram

Accuracy analysis of three results is performed on the aerial triangulation under different control point layout conditions, and the results are shown in the table below.

\begin{tabular}{|c|c|c|c|c|c|c|c|c|c|}
\hline \multirow[b]{2}{*}{ No } & \multicolumn{3}{|c|}{$700 \mathrm{~m}$ span } & \multicolumn{3}{|c|}{$500 \mathrm{~m}$ span } & \multicolumn{3}{|c|}{$250 \mathrm{~m}$ span } \\
\hline & $\begin{array}{l}X \\
\mathrm{~cm}\end{array}$ & $\begin{array}{l}\mathrm{Y} \\
\mathrm{cm}\end{array}$ & $\begin{array}{l}\mathrm{Z} \\
\mathrm{cm}\end{array}$ & $\begin{array}{l}X \\
\mathrm{~cm}\end{array}$ & $\begin{array}{l}\mathrm{Y} \\
\mathrm{cm}\end{array}$ & $\begin{array}{l}\mathrm{Z} \\
\mathrm{cm}\end{array}$ & $\begin{array}{l}X \\
\mathrm{~cm}\end{array}$ & $\begin{array}{l}\mathrm{Y} \\
\mathrm{cm}\end{array}$ & $\begin{array}{l}\mathrm{Z} \\
\mathrm{cm}\end{array}$ \\
\hline J067 & 17 & -3 & -66 & -3 & -5 & 28 & 1 & -11 & 1 \\
\hline J068 & 4 & 9 & 1 & -7 & 2 & 1 & -9 & 3 & -1 \\
\hline J069 & 6 & 27 & 28 & 0 & 4 & 0 & 1 & 3 & -2 \\
\hline J070 & 21 & 44 & 27 & 11 & -4 & 17 & 7 & 3 & 6 \\
\hline J075 & 4 & 35 & -10 & 1 & 10 & 34 & -4 & 7 & 15 \\
\hline J076 & 33 & 3 & -26 & -7 & 4 & 25 & -12 & 0 & 7 \\
\hline $\mathrm{J} 130$ & 0 & 3 & -67 & -15 & 10 & 19 & -8 & 3 & -3 \\
\hline J139 & 10 & 0 & -16 & -5 & 3 & 17 & 0 & 3 & -5 \\
\hline J141 & 9 & -9 & 13 & 1 & 2 & 1 & 3 & 1 & -8 \\
\hline $\mathrm{J} 142$ & -1 & -13 & 16 & 5 & 2 & 5 & 7 & 5 & -5 \\
\hline $\mathrm{J} 143$ & -11 & -12 & 3 & 0 & 3 & 0 & 4 & 1 & -6 \\
\hline J144 & 10 & 13 & -18 & -14 & -8 & -5 & -13 & -4 & -6 \\
\hline J145 & 11 & -3 & 16 & -10 & 6 & -14 & -8 & 6 & -8 \\
\hline J146 & 17 & 22 & -39 & -12 & -14 & 10 & -12 & -12 & 7 \\
\hline $\mathrm{j} 218$ & 5 & 12 & -10 & 3 & -6 & 4 & 0 & -5 & 3 \\
\hline $\mathrm{j} 219$ & 14 & 12 & 2 & -6 & -6 & 9 & -9 & -3 & 13 \\
\hline $\mathrm{j} 220$ & 23 & 19 & 3 & -11 & -10 & 3 & -11 & -5 & 3 \\
\hline $\mathrm{j} 221$ & 3 & 5 & 40 & 8 & 1 & -17 & 2 & 2 & -8 \\
\hline $\mathrm{j} 222$ & 6 & 1 & 27 & 4 & -3 & -13 & -1 & -1 & -2 \\
\hline $\mathrm{j} 223$ & 6 & -1 & 32 & -1 & 1 & -14 & -2 & 0 & -2 \\
\hline $\mathrm{j} 224$ & 21 & 21 & 36 & 5 & -6 & -4 & 2 & -5 & -3 \\
\hline $\mathrm{j} 225$ & 23 & 12 & 6 & 2 & 0 & 3 & -1 & 1 & 2 \\
\hline $\mathrm{j} 226$ & 0 & -5 & 6 & 8 & 2 & -3 & 4 & 2 & 2 \\
\hline $\mathrm{j} 227$ & -5 & -7 & 0 & 4 & 3 & 8 & 0 & 4 & 7 \\
\hline $\mathrm{j} 228$ & 1 & 5 & 33 & 3 & -6 & -14 & 4 & -3 & -4 \\
\hline $\begin{array}{l}\text { Mean } \\
\text { Squ- } \\
\text { are } \\
\text { error }\end{array}$ & 22.4 & & 28.1 & 9.3 & & 13.9 & 8 & & 6.2 \\
\hline
\end{tabular}

Table 1. Precision Table

From the residual statistics of check points, if $700 \mathrm{~m}$ is used as the control point for the placement of the unit, the plane mean square error can reach $22.4 \mathrm{~cm}$ and the elevation accuracy can reach $28.1 \mathrm{~cm}$; if $500 \mathrm{~m}$ is used as the control point to lay the unit. the plane mean square error can reach $9.3 \mathrm{~cm}$ and the elevation accuracy can reach $13.9 \mathrm{~cm}$; if a unit of $250 \mathrm{~m}$ is used as the control point, the plane mean square error can reach $8.0 \mathrm{~cm}$ and the elevation accuracy can reach $6.2 \mathrm{~cm}$. From the statistic results, the layout density of the control points has certain influence on the precision of the aerial triangulation of oblique photography. Within a certain range, the density of control points is densely packed. The precision is higher, and the influence on the elevation accuracy is greater.

\subsection{Influence of the Precision of Control Points on the Precision of Aerial Triangulation of Oblique Photography}

At present, the main methods of the layout of control points are the classical GPS static relative positioning measurement method and network RTK (Yang Yongping, Hong Shaoming, Duan Delei, 2013) measurement method. Network RTK (Zhao Qingzhi, Zhang Shubi,2012) technology refers to the GNSS base station network, base station site, and data communication network that are based on a number of precise geodetic coordinates. The real-time data processing center forms a GNSS continuous operation base station network to obtain real-time accurate spatial coordinate results. The client sends the approximate position observation data through the GPRS network, and the data station network provides the centimeter precision positioning results in real time computation and finally transmits the network RTK measurement results back to the user. The plane accuracy can generally reach $2 \mathrm{~cm}$, and the elevation accuracy can generally reach $5 \mathrm{~cm}$. The classical GPS static relative positioning method is to set up more than three GPS receivers synchronously on the fixed ground sign points, and measure the GNSS control points with precise known results in parallel, to complete the calculation and adjustment of the spatial baseline network through the GPS data processing software, and to integrate the ground marking points into the known coordinate system, and provides centimeter-level precision control network results for data post-processing finally. The plane accuracy can generally reach $1 \mathrm{~cm}$, and the elevation accuracy can generally reach $2 \mathrm{~cm}$. It can be seen from the observation method that the data acquisition efficiency of network RTK method is high, and the precision can meet the requirement of the control points of aerial survey. Therefore, in the general photogrammetry project, the network RTK method is usually used for control point measurement in the CORS signal receivable area.

In practice, we simulate the influence of the accuracy of control points on the precision of aerial triangulation by simulating different precision control points. One control point source is the data obtained by the network RTK, and the other one is the feature point collected on the 3D model whose plane accuracy can reach $5 \mathrm{~cm}$ and the elevation accuracy can reach $10 \mathrm{~cm}$. We compare the precision of the results using the two kinds of control points with same position in aerial triangulation. We come to the conclusion that if the image resolution is between the two control points precision, then the control point has great effect on the precision of aerial triangulation results, and on the basis of original results, the precision of aerial triangulation will usually reduce the level of image resolution. If the accuracy of the control points is higher than the image resolution, that is, the accuracy of the control points is sub-pixel, then the difference of the accuracy of the control points has little effect on the precision of aerial triangulation. 


\section{SUMMARY AND OUTLOOK}

In this paper, the influence of density and precision of control points layout on aerial triangulation is analyzed theoretically and practically. Although the experiment and experimental data in this paper are not in the ideal state, the data source is obtained from the actual production. For example, control point density can achieve a smaller interval and the precision of the low-level control points is simulated. However, the corresponding conclusion is verified in many practical projects, so the research of this paper can provide some theoretical and practical guidance for the practical project of oblique photography under the circumstance that there is no corresponding specification as the guidance for data processing of oblique photography at present.

\section{REFERENCES}

Deng Fang, Zhang Jun, etc.2011.On Distribution Scheme of Photo Control Points for UCD/UCX Aerial Imagery. Bulletin of Surveying and Mapping, 8(2), pp. 9-12.

Fan Panfeng, Li Lulu,2017. A Three-dimensional Modeling Study Based on the Technique of Low-altitude UAV Oblique Photogrammetry and Smart3D Software. Bulletin of Surveying and Mapping, S2, pp.77-81.

Wang Jing, etc.2011. 3D Model Reconstruction and Precision Analysis for Oblique Photogrammetry Based on UVA. Standard of Surveying and Mapping,33(3), pp. 32-35.

Yang Yongping, Hong Shaoming, Duan Delei, 2013.Application of GPS RTK Position Technique in Digitalized Mapping. Railway Investigation and Surveying,24(2), pp. 10-13.

Zhao Qingzhi, Zhang Shubi,2012. The Exploration and Application of control measures of GPS Static Relative Positioning Techniques in Urban Areas. GNSS World of China, 37(4), pp.64-66. 